\title{
Towards Understanding Tuberculosis- Related Issues in North Korea: A Narrative Review of North Korean Literature
}

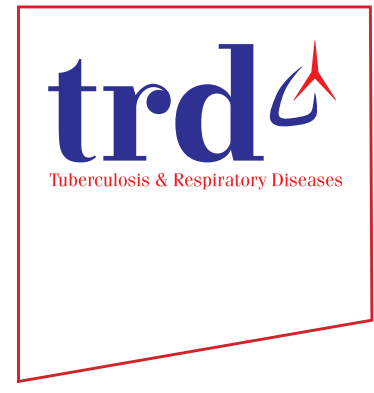

\author{
Chang-Jun Lee ${ }^{1, *(\mathbb{D}}$, Sungwhan Lee ${ }^{1, *(\mathbb{D}}$, Hee-Jin Kim, M.D., M.P.H. ${ }^{2}$ and Young Ae Kang, \\ M.D., Ph.D. ${ }^{3}$ (1) \\ ${ }^{1}$ Yonsei University College of Medicine, Seoul, ${ }^{2}$ Central Training Institute, Korean National Tuberculosis Association, Seoul, \\ ${ }^{3}$ Division of Pulmonology, Department of Internal Medicine, Yonsei University College of Medicine, Seoul, Korea
}

Background: North Korea is one of the 30 countries with the highest tuberculosis (TB) and drug-resistant TB burdened. To understand the medical issues and research trends associated with TB in North Korea, we performed a comprehensive review of articles related to clinical and laboratory research on TB published in North Korean medical journals.

Methods: We reviewed all types of TB-related articles published in nine North Korean medical journals (Yebang ŭihak: Preventive medicine; Koryo ŭihak: Korea Medicine; Chosŏn ŭihak: Chosun Medicine; Naekwa: Internal Medicine; Soa, sanbuinkwa: Pediatrics, Obstetrics, and Gynecology, Surgery; Ǔihak: Medicine; Kich'o ŭihak: Basic Medicine; and Chosŏn yakhak: Chosun Pharmacy). We classified the articles according to the type and field of study and analyzed the data qualitatively to gain insights.

Results: We reviewed 106 articles (one- or two-page length) written in Korean, including reviews (n=43), original articles $(n=52)$, and case reports $(n=8)$. They were classified as follows: articles on diagnosis $(n=52,49 \%)$ and treatment $(n=39$, 37\%). None of the studies investigated the commercialized molecular diagnosis systems such as Xpert MTB/RIF. Directly Observed Treatment, Short-course was reported as the basic treatment approach. Furthermore, six studies used Korean traditional medicines for treating TB, with one of them containing snake venom.

Conclusion: The articles were not sufficiently detailed. Original articles on the treatment of multi-drug resistant TB were not found, and those on latent tuberculosis infection and nontuberculous mycobacteria were limited. To understand the current medical issues associated with TB in North Korea, articles from these nine journals were not sufficient.

Keywords: Tuberculosis; Democratic People's Republic of Korea; Review

Address for correspondence: Young Ae Kang, M.D., Ph.D.

Division of Pulmonology, Department of Internal Medicine, Yonsei University College of Medicine, 50-1 Yonsei-ro, Seodaemun-gu, Seoul 03722, Korea

Phone: 82-2-2228-1954, Fax: 82-2-393-6884

E-mail: mdkang@yuhs.ac

${ }^{*}$ Chang-Jun Lee and Sungwhan Lee contributed equally to this work.

Received: Sep. 26, 2019

Revised: Jan. 28, 2020

Accepted: Feb. 25, 2020

Published online: Mar. 10, 2020

(c) It is identical to the Creative Commons Attribution Non-Commercial License (http://creativecommons.org/licenses/by-nc/4.0/).

Copyright $\subset 2020$

\section{Introduction}

Despite efforts from international communities to support the North Korean tuberculosis (TB) control program, TB is one of the major infectious diseases in North Korea. Lower respiratory tract infections, TB and diarrheal diseases are the major infectious diseases in North Korea. North Korea is one of the 30 countries with the highest burden of $\mathrm{TB}$ and rifampin resistant (RR)/multi-drug resistant (MDR)-TB. According to the World Health Organization (WHO), estimated incidence rate of TB has increased from 345/100,000 in 2010 to $513 / 100,000$ in 2018 and estimated incidence of RR/MDR-TB in 2018 was $5200(20 / 100,000)^{1,2}$. North Korea reported 1,782 cases of laboratory-confirmed RR/MDR-TB with initiation of 
treatment in 1,487 patient in $2018^{1}$.

The North Korean health care system has been devastated by natural disasters and the loss of support occasioned by the break-up of the Soviet Union since mid-1990s, making it difficult to respond to poverty- and nutrition-related diseases such as $\mathrm{TB}^{3,4}$. In addition, the tight United Nations sanctions due to the nuclear program of North Korea have made it difficult to maintain the humanitarian assistance operations for the management of TB and MDR-TB in North Korea ${ }^{5}$.

After being divided into separate countries 71 years ago, South Korea and North Korea have shown differences in many health-related statistics ${ }^{6}$, especially with regard to TB. Recently, South Korea and North Korea have initiated discussions about a joint response system for fighting contagious disease, such as TB and malaria ${ }^{7}$; however, data on the incidence and management of TB and MDR-TB in North Korea are scarce. Because of the lack of primary source of data and samples, many South Korean researchers have estimated the circumstances in North Korea on the basis of empirical data collected through testimonies or survey results from North Korean defectors ${ }^{8}$; however, generalizability of these data is limited by the methodology.

Thus, researchers have tried to investigate trends of research on TB or other diseases using North Korean medical journals to supplement for the lack of primary data and to understand the current disease status in North Korea ${ }^{9}$. The analysis of research published in medical journals can provide insights into the current status of research by providing information on the academic interests of a society and on the level of medical development based on the use of technology.

In South Korea, nine medical journals from North Korea are available. This study aimed to perform a comprehensive review of articles related to clinical and laboratory research on TB in North Korean medical journals to understand the medial issues and research trends on the basis of available data.

\section{Materials and Methods}

In South Korea, nine academic journals published by North Korean organizations are available in the Information Center on North Korea maintained by the Ministry of Unification. Researchers can access these journals at the center. The following is the list of these nine medical journals: Yebang ŭihak (Preventive Medicine), Koryo ŭihak (Korea Medicine), Chosŏn ŭihak (Chosun Medicine), Naekwa (Internal Medicine), Soa, sanbuinkwa (Pediatrics, Obstetrics, and Gynecology, Surgery), UUihak (Medicine), Kich'o ŭihak (Basic Medicine), and Chosŏn yakhak (Chosun Pharmacy). We analyzed the articles published from 2006 to 2018 in all these journals, with the exception of two journals, Üihak (Medicine) and Koryo ŭihak (Korea Medicine). In Ǔihak (Medicine) and Koryo ŭihak (Korea Medicine), only articles published during the periods of 2006-2008 and 2016-2018, respectively, were available. Because this study analyzed previously published literature, approval from the institutional review board was not required.

\section{Search strategies and data collection}

We screened all articles in the nine medical journals to identify those related to TB. All types of articles, except "Introduction of Journal" and "News of Academy," were included from each journal. Two independent authors (C.J. Lee and S. Lee) initially screened the articles by titles to identify those related to TB; they subsequently assessed the full text of the articles that were preliminarily identified to be relevant. No restrictions were applied regarding study design, patient number, methods of data collection, or article type (case reports, review papers, clinical trials, and basic laboratory research).

\section{Data extraction and analysis}

Names of authors and journals, titles, and year of publication corresponding to all the included articles were recorded in a Microsoft Excel (Microsoft Corp., Redmond, WA, USA) file. We analyzed the data in included articles on the basis of the classification of disease and field of study. Two researchers (C.J. Lee and S. Lee) conducted all research data extraction and classification, and when differing opinions were presented, consensus was reached by discussion.

\section{Results}

There were 18,596 articles in those nine journals. After title-based screening and assessment of full-text articles, 106 TB-related articles $(0.6 \%)$ were included in the final analysis (Figure 1). Among these 106 articles, 75 articles were from two journals: Yebang ŭihak (Preventive Medicine), 48 articles (45.3\%); Naekwa (Internal Medicine), 27 articles (25.5\%). After excluding two journals, Ǔihak (Medicine) and Koryo ŭihak (Korea Medicine), the number of published articles on TB showed an increasing trend from 2008 to 2015, with the highest number of articles $(n=16)$ published in 2015. All the articles $(n=106)$ were one or two pages in length and were written in Korean. Seven articles published in Chosŏn ŭihak

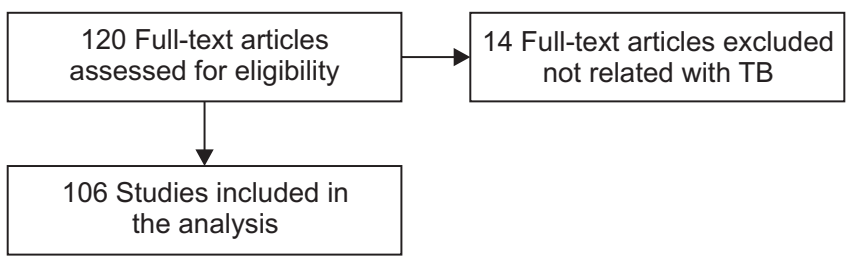

Figure 1. Flowchart describing the selection of articles included in the analysis. TB: tuberculosis. 
(Chosun Medicine) and one articles published in Naekwa (Internal Medicine) had a 2-3 sentence-long summary in English. The articles typically had two authors (74 out of 106 articles) without the contact information of corresponding author and less than five references which were at the end of the article without in-text citation. Articles usually began with citation of the Supreme leaders' instruction and reflection of their ideological views and ideas unique to North Korea ${ }^{10}$. In the articles, tables, graphs, and images were partially illustrated by hand, and some statistics such as percentages and pvalues were presented with no specific statistical method. All the experimental and interventional articles showed positive results, and there was no mention of ethical approval for human study.

\section{Classification based on field}

The articles $(\mathrm{n}=106)$ were classified as follows: reviews $(n=43)$, original articles $(n=52)$, and case reports $(n=8)$. Among the 106 articles, 90 involved human studies and 16 involved animal studies. On the basis of the field of study, the articles were classified as follows: articles on diagnosis, 52 (49\%); treat-

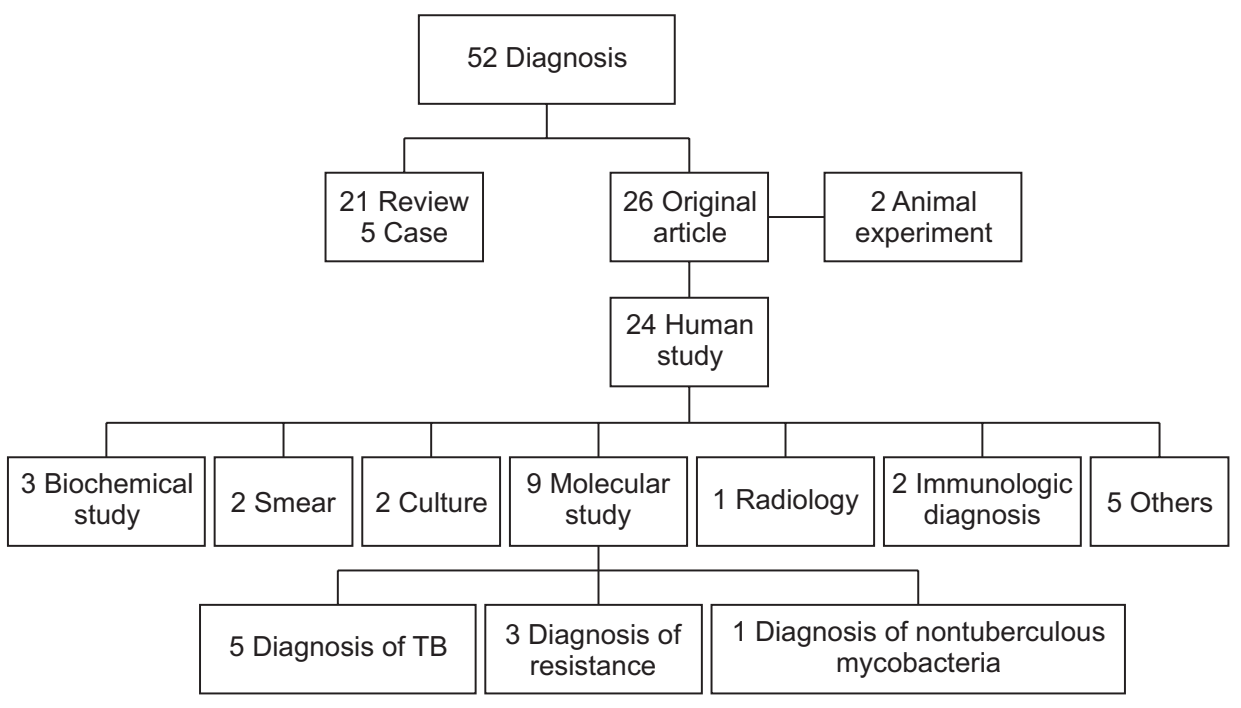

39 Treatment
Figure 2. Classification of articles related to the "Diagnosis of TB." TB: tuberculosis.

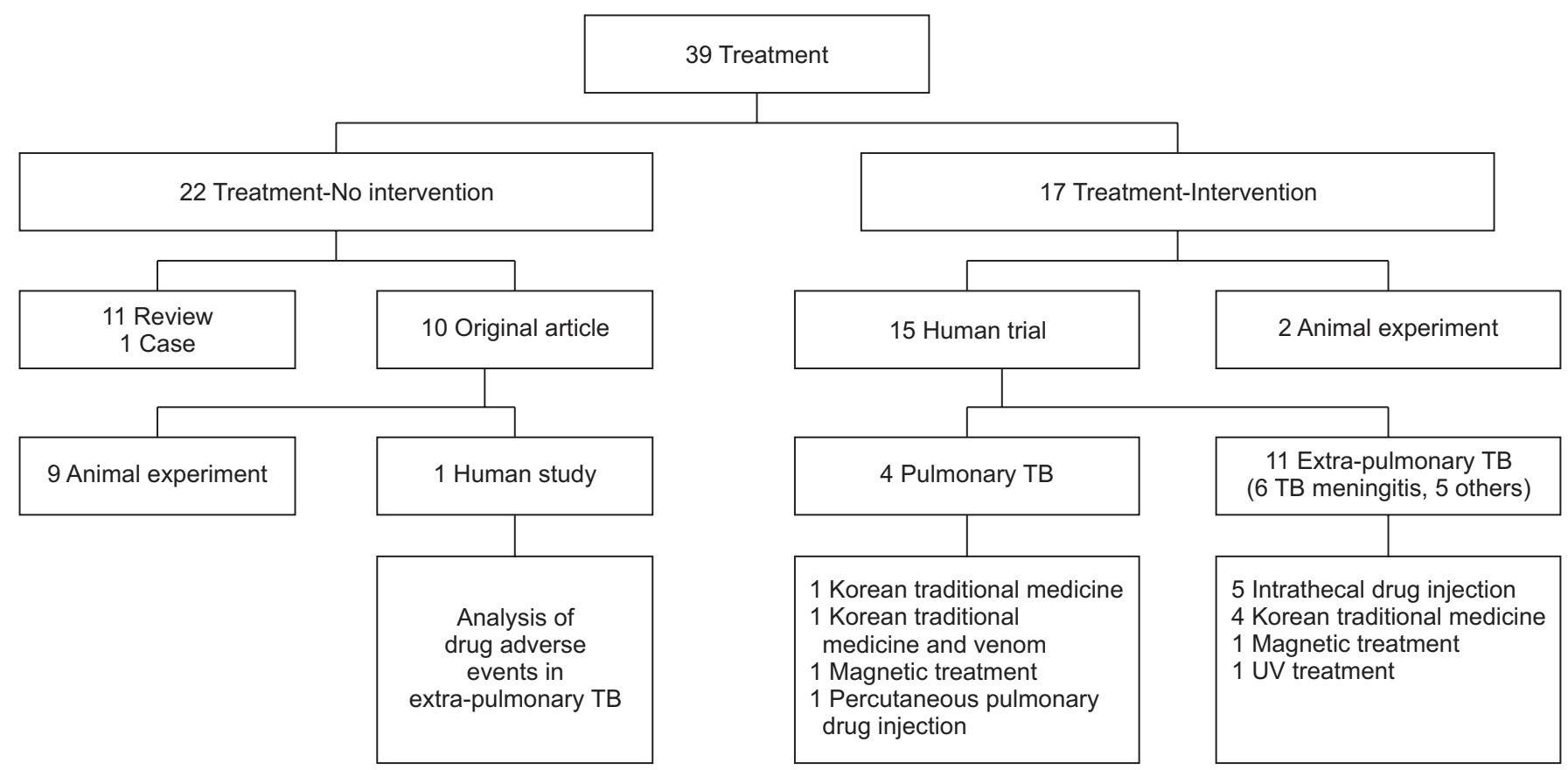

Figure 3. Classification of articles related to the "Treatment of TB." TB: tuberculosis; UV: ultraviolet. 

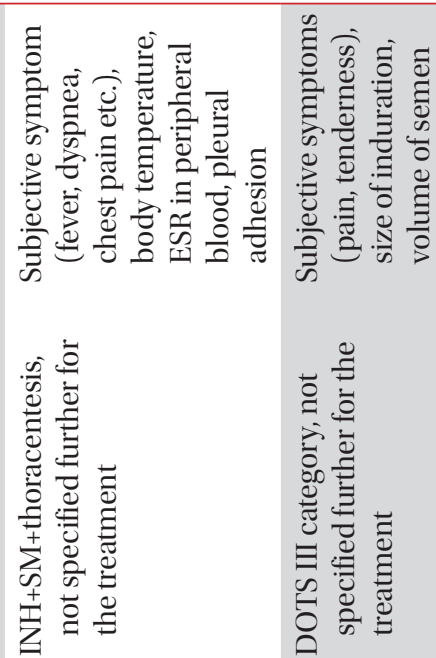

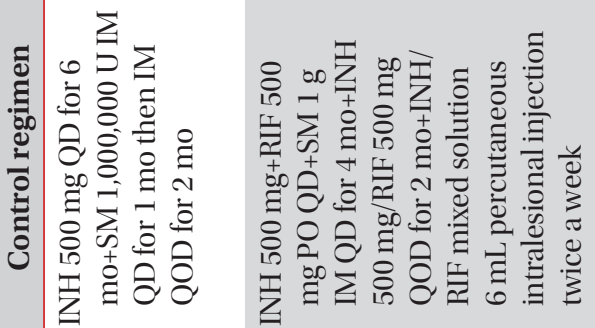
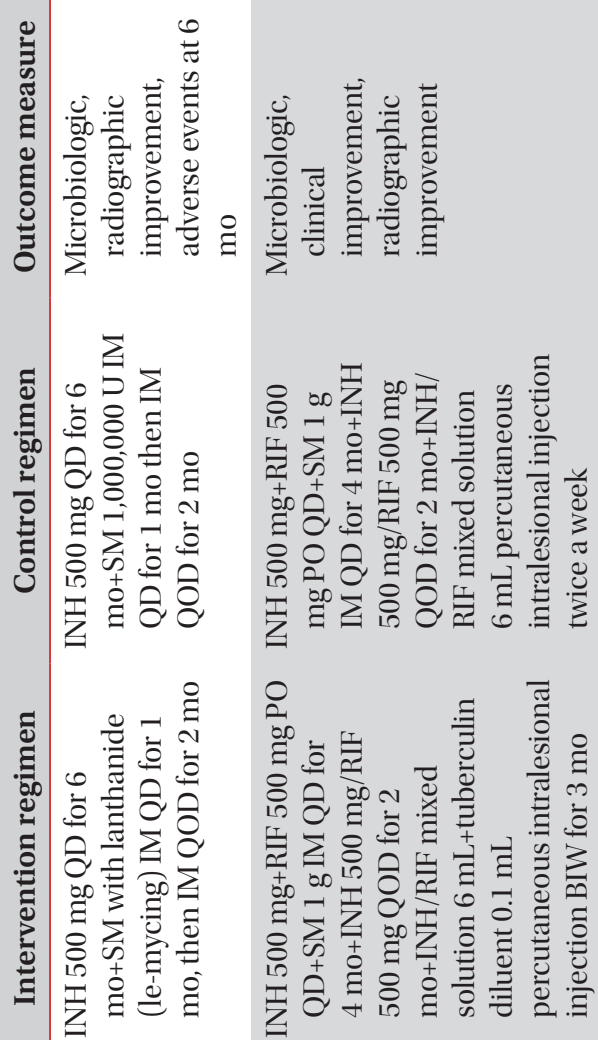

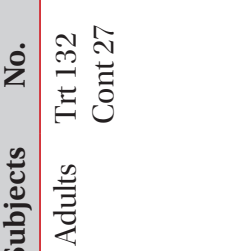

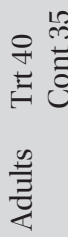

है

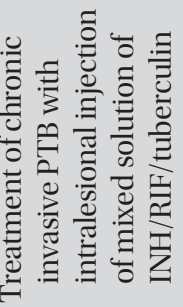

๕ั้

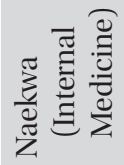

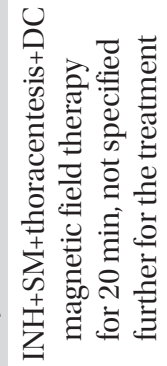

占

$\frac{n}{\frac{Z}{2}}$

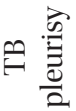

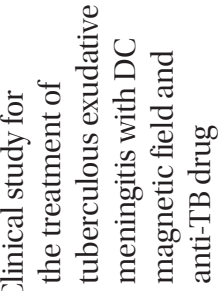

๕ั่

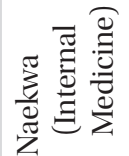
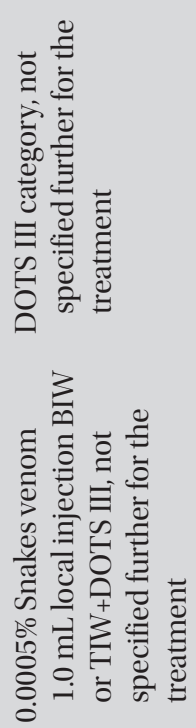

咅

$\frac{n}{\frac{3}{2}}$

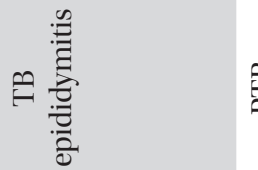

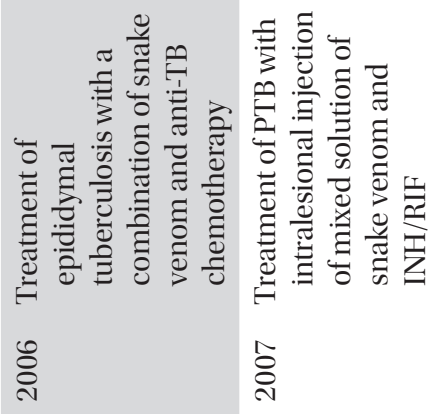

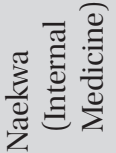

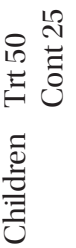

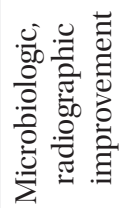

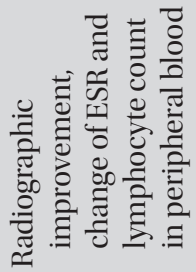

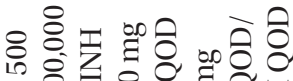

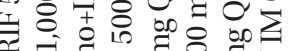

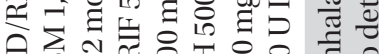

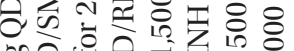

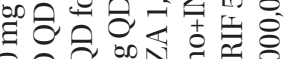

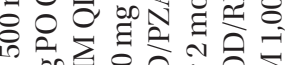

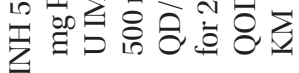
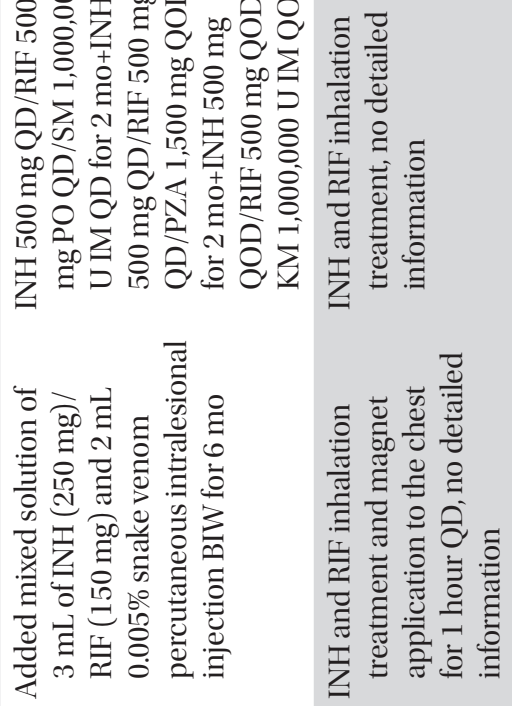

$10 \stackrel{10}{=}$

ฮี

$\frac{\operatorname{tg}}{\overline{3}}$

قै

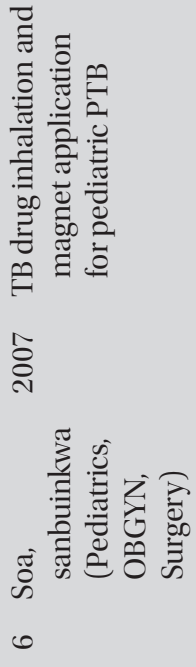




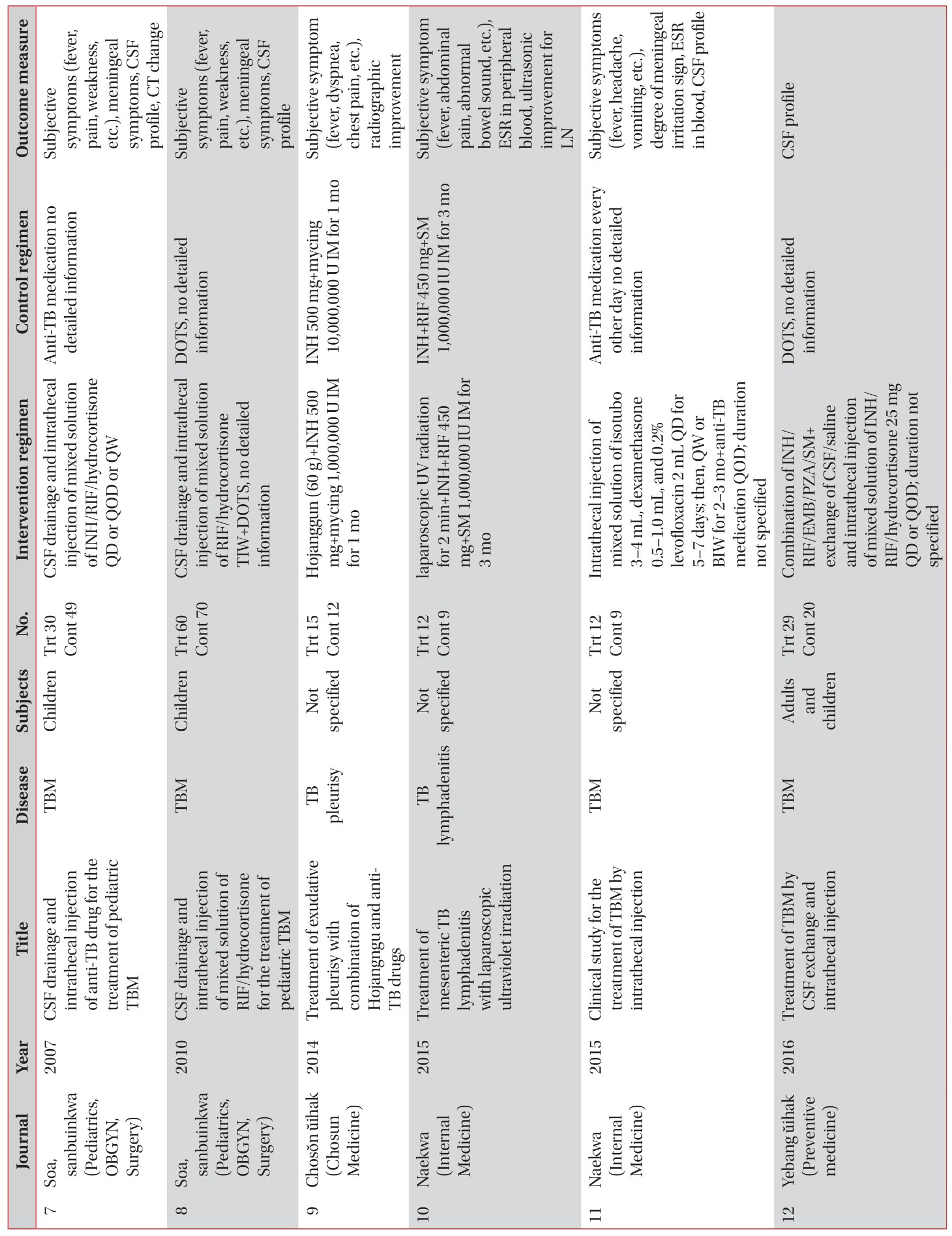




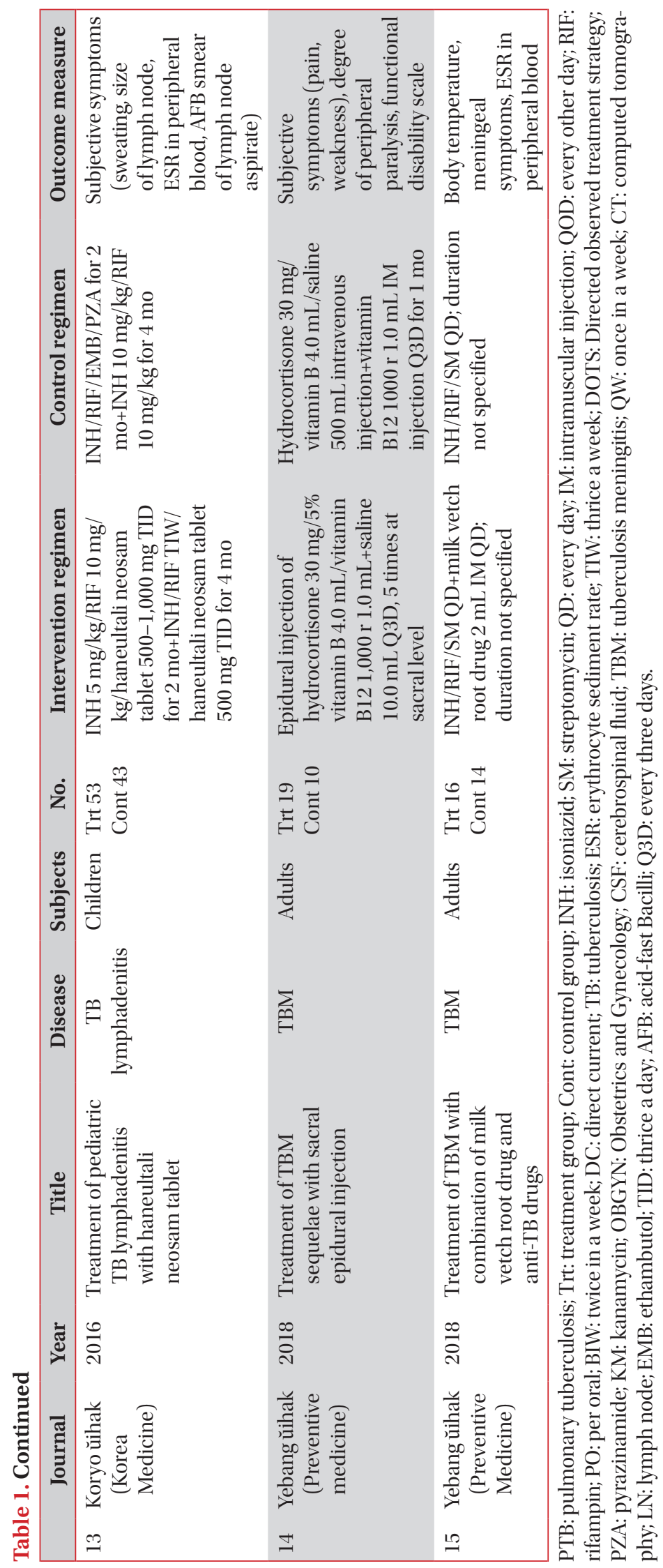


ment, 39 (37\%); epidemiology, 3 (3\%); pathogenesis, 2 (2\%); vaccines, 3 (3\%); diagnosis+treatment, 5 (5\%); and others, 2 (2\%).

Among the 52 articles on the diagnosis of TB, 26 were original articles with 24 involving human subjects (Figure 2). These 24 articles corresponded to different methods of TB diagnosis: molecular diagnosis $(n=9)$, biochemical diagnosis $(n=3)$, acidfast Bacilli (AFB) smear-based diagnosis $(n=2)$, mycobacterial culture-based diagnosis $(n=2)$, immunologic diagnosis $(n=2)$, and radiological diagnosis $(n=1)$. Among the nine articles on molecular diagnosis, five corresponded to molecular diagnosis of TB, three corresponded to molecular diagnosis of drug-resistant $\mathrm{TB}$, and one corresponded to the diagnosis of nontuberculous mycobacteria (NTM). None of the studies investigated any aspect of the commercialized molecular diagnosis systems such as Gene Xpert MTB/RIF assay (Cepheid, Sunnyvale, CA, USA) or GenoType MTBDRplus assay (Hain Lifescience, Nehren, Germany). In addition, there was no detailed description of molecular diagnostic procedures such as polymerase chain reaction or microarray analysis used for the detection of TB or $r p o B$ gene mutation.

Among the 39 articles on the treatment of TB, 17 corresponded to interventional trials (Figure 3). Among these 17 interventional studies, four and 11 articles were related to the treatment of pulmonary TB and extra-pulmonary TB, respectively, in humans. In Table 1, the detailed treatment regimens for 15 human trials are summarized. The non-standardized regimen and intermittent treatment were common in these 15 trials. In six studies, Korean traditional medicine was used for the treatment of TB; one of these medicines involved the use of snake venom. There were no studies on interventional treatment for drug-resistant TB.

\section{Classification based on topic}

We found 13 articles on drug resistant TB, two articles/ year in 2007, 2012, 2013, 2014, 2015, and 2017 and one in 2018. Among them, five were reviews and eight were original articles. A review article published in 2013 summarized the "Guidelines for the programmatic management of drugresistant TB, $2008 \mathrm{WHO}^{\text {"11 }}$, and a review article published in 2014 mentioned about Gene Xpert/MTB RIF assay. In the latter review, the author mentioned Xpert MTB/RIF assay and nitrate reductase assay for rapid drug susceptibility test for TB; they discussed more positive points of views on the nitrated reductase assay. All the original articles on drug resistant TB in humans were about the diagnosis of drug resistance: molecular diagnosis $(n=3)$, smear morphology-based diagnosis $(n=1)$, biochemical assay-based diagnosis $(n=1)$, and culturebased drug susceptibility test-based diagnosis $(n=1)$ (Figure 4). In one study involving analysis of the frequency of drug resistance using a culture-based drug susceptibility test, the authors assessed the resistance of 246 culture isolates to isoniazid (INH), streptomycin (SM), rifampin (RIF), ethambutol (EMB), and pyrazinamide (PZA). Among 33 treatment naïve TB isolates, five (15.1\%) showed resistance to INH and six (18.1\%) showed resistance to RIF according to the report. Among 53 isolates after over 6 months of treatment, 47 (88.6\%) were resistant to INH and 36 (67.9\%) were resistant to $\mathrm{RIF}^{12}$.

One review article and one case report were about latent tuberculosis infection (LTBI). In the case report, the authors diagnosed LTBI using tuberculin skin test (30 mm of induration) and QuantiFERON TB Gold 2G (Cellestis, Melbourne, Australia) in a female patient with systematic lupus erythematosus. There were four articles on the molecular diagnosis of NTM: two case reports, one review, and one original article.

Among the 106 articles, 37 articles (34.6\%) covered extrapulmonary TB. TB meningitis was the most common form of

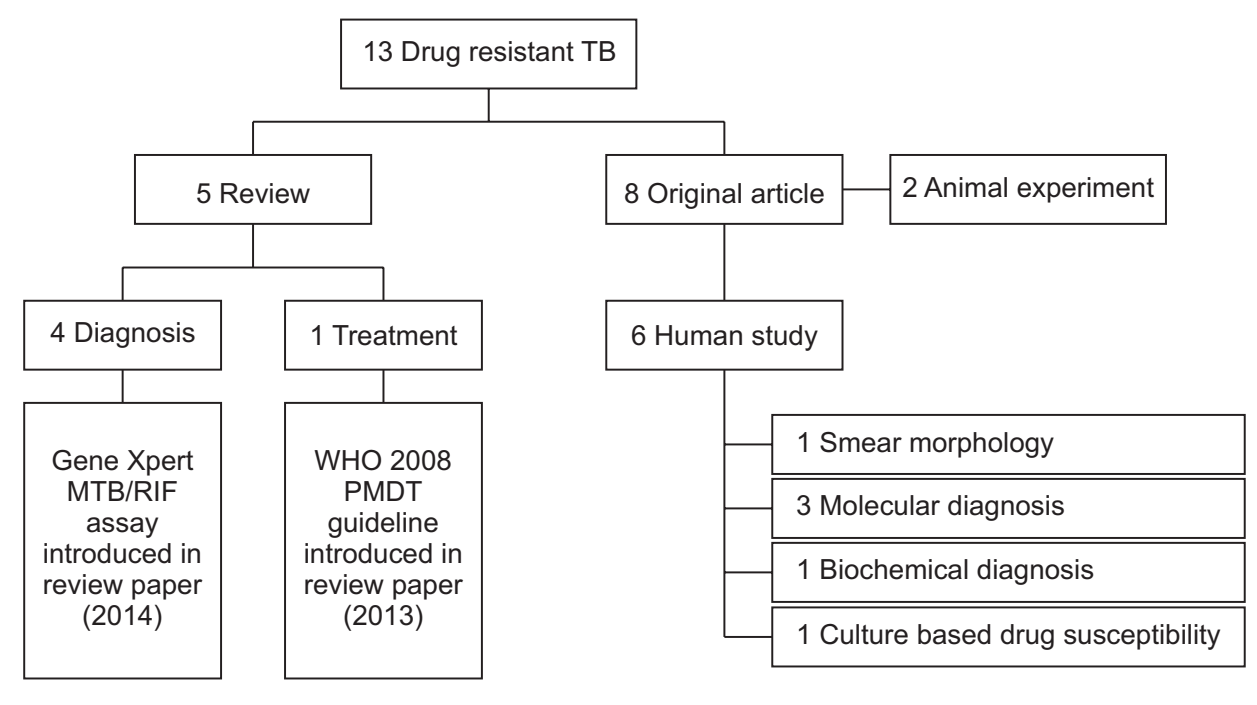

Figure 4. Classification of articles related to "Drug resistant TB." TB: tuberculosis; WHO: World Health Organization; PMDT: programmatic management of drug resistant tuberculosis. 
extra-pulmonary TB (18 articles, 49\%). Especially, five studies focused on investigating the effect of intrathecal anti-TB drug injection for the treatment of TB meningitis and four studies focused on investigating the synergistic effect of anti-TB medicines and traditional Korean medicines for the treatment of extra-pulmonary TB (Table 1). In these studies, the additional effect of traditional medicine in relieving meningitis symptoms or improving treatment outcome of pleural TB and epididymal TB were assessed.

\section{Diagnosis and treatment of TB in North Korea}

According to a review article, AFB smear is the mainstay for diagnosing TB and monitoring treatment response in North Korea. In one paper published in 2007, the author emphasized that AFB smear should be performed at least 3 times for the diagnosis of TB and that when the AFB smear is negative, TB could be diagnosed by imaging-based studies and expert opinion. For the treatment of TB, Directly Observed Treatment, Short-course (DOTS) was described as a basic approach and INH, RIF, EMB, PZA, and SM were mentioned as the drugs used. Category I and II TB treatment regimens were the standard treatment regimens used.

\section{Discussion}

We analyzed 106 articles dealing with TB in North Korea. This can be a starting point for evaluating the status of TB burden and research infrastructure in North Korea ${ }^{13}$.

Articles on TB have been increasing since 2010. About half of these articles were published in one journal, Yebang ŭihak (Preventive Medicine); this possibly implies the importance of prevention, with regard to public health-related aspects of $\mathrm{TB}$ in North Korea.

For the diagnosis of TB, research on new technologiessuch as molecular diagnostics-has increased since 2012. Studies focusing on the molecular diagnosis of resistance have also been conducted, but no research was conducted on commercialized kits that can be used for actual patient care. In particular, studies on Gene Xpert MTB/RIF assay, which is highly recommended for the rapid diagnosis of TB and MDR$\mathrm{TB}$ by $\mathrm{WHO}^{2,14}$, have not been reported in any of the analyzed articles. This could be a result of relatively recent introduction of Gene Xpert MTB/RIF in North Korea. According to the Joint Monitoring Mission Tuberculosis Control Programme ${ }^{15}$ report, GeneXpert system was first set up in North Korea in the national reference laboratory in 2013 and 2016. Four more GeneXpert machines were procured for provincial level laboratories under Global Fund support. However, a shortage of cartridges is hindering the use of these machines ${ }^{15}$.

The proportion of research focused on culture-based diagnosis of TB and drug resistance was also small, which could indicate that it was difficult to establish a proper culture system in North Korea. The drug susceptibility test for TB was performed in the National TB Reference laboratory in Pyongyang. An effort to build a National TB reference laboratory with facilities for diagnosing drug resistant TB in North Korea was led by the Bay Area TB Consortium, a network of experts from Stanford university, state's TB program, and Christian Friends of Korea in $2009^{16-18}$. However, the maintenance and actual capacity of that laboratory might currently be undersupported because of economic sanctions from the UN. This suggests the insufficiency of infrastructure and resources of a national program for the diagnosis and treatment of drugresistant TB in North Korea.

For the treatment of TB, the Ministry of Public Health (MOPH) of North Korea adopted DOTS, the WHO-recommended approach for TB control in resource-limited setting in $1998^{4}$. North Korea began procurement of TB drugs from the Global Drug Facility, a WHO-led initiative that has supplied quality assured drugs to over 90 countries from 2003. In 2010, the Global Fund to Fight AIDS, TB, and Malaria (GFATM) began a major project to strengthen TB control in North Korea, and this investment enabled the national TB program to undergo a much-needed modernization, including the introduction of new diagnostic and treatment protocols ${ }^{19}$. However, a small portion of this international aid was allocated for the treatment of drug-resistant $\mathrm{TB}^{3}$. The MOPH of North Korea first began diagnosing and treating MDR-TB in 2008 with support from the Eugene Bell Foundation (EBF), a US- and South Korea-based nongovernmental organization ${ }^{20}$. However, this EBF program did not cover North Korea in entirety.

Based on the analysis of articles on TB from the nine North Korean journals, the procurement of first line TB drugs was estimated to be insufficient. Although category I standard regimen with daily INH/RIF/EMB/PZA administration for 2 months and daily INH/RIF administration for 4 months for new smear positive or extensive smear negative pulmonary TB and DOTS for severe forms of extra-pulmonary TB appear to be similar to the basic treatment prescription, in some studies, patients were treated with 3 drugs-INH, RIF and SM or PZA-every other day. Intermittent treatment, which is no longer recommended by WHO, is still provided in the continuation phase for drug-sensitive TB in North Korea, according to the articles. In addition, category II treatment is still in use with SM; however, this regimen is contra-indicated by $\mathrm{WHO}^{15}$. In other studies, patients were treated with two antiTB medicines combined with Korean traditional medicine for 2 or 3 months. Five articles investigated the effects of Korean traditional medicine for the treatment of TB. This may imply two things: (1) North Korea is continuing its efforts to develop new treatments for TB using Korean traditional medicines and (2) the lack of reliable supply of anti-TB drugs has led to exploration of traditional options.

With regard to the prevalence of drug resistance, consider- 
able proportion of primary resistance to INH and RIF in new treatment ( $15.1 \%$ for INH, $18.1 \%$ for RIF) was reported in one article $^{12}$. These data are quite different from the drug resistance data published in the WHO report, that is, an estimated percentage of MDR/RR-TB in new cases was $2.2 \%$ in $2018^{1}$. Thus, we could not conclude about the presence of high resistance in new TB patients in North Korea based on one selected report.

We could not find any studies on the treatment of MDR$\mathrm{TB}$ in the nine North Korean journals. According to previous reports, the EBF enrolled over 1,000 patients with MDR-TB in one year for treatment with second line anti-TB drugs; however, it constituted a small proportion of the total burden of disease ${ }^{20}$ when we considered the estimated incidence rate of RR/MDR-TB, $5200(20 / 100,000)^{1}$ in 2018. Because the treatment of MDR-TB in North Korea is dependent on international aids from organizations such as EBF and GFATM, there seems to be insufficient domestic research on this aspect.

This study has several limitations. First, this study focused on a quantitative analysis rather than content analysis because of the lack of detailed information in most of the included articles. Second, our analysis was limited to articles available between 2006 and 2018. Thus, it was difficult to perform time series analysis. In addition, we did not include representative TB reports from North Korea such as the WHO report or TB prevalence survey results.

Despite these limitations, this study was able to provide indirect evidence regarding the current conditions of diagnosis and treatment of TB and research infrastructure in North Korea, which is especially useful because direct investigation of burden of TB in North Korea is difficult, and currently, limited data exist.

In conclusion, articles on TB have been published steadily in North Korea. However, the articles were quite shorter than the standard article length recommended by international journals. Articles on the treatment of MDR-TB were not found. LTBI and NTM were not major topics in North Korean articles. To understand the current situation of TB in North Korea, further analysis by including English papers from international journals and other epidemiological reports from international organizations will be helpful.

\section{Authors' Contributions}

Conceptualization: Kang YA, Kim HJ. Formal analysis: Lee CJ, Lee S, Kim HJ, Kang YA. Data curation: Lee CJ, Lee S. Writing - original draft: Lee CJ, Lee S, Kang YA. Writing - review \& editing: Lee CJ, Lee S, Kim HJ, Kang YA.

\section{Conflicts of Interest}

No potential conflict of interest relevant to this article was reported.

\section{Acknowledgments}

We thank Woo Taek Jeon, MD, PhD (Department of Psychiatry and Institute of Behavioral Science in Medicine, Yonsei University College of Medicine), Dr. Hye-won Kim (Yonsei University Education center) for inspiration in this research.

\section{Funding}

The present study was supported under the framework of the international cooperation program managed by the National Research Foundation of Korea (2019K1A5A2077463, FY2019). The funding source had no role in the study process, including the design, sample collection, analysis, or interpretation of the results.

\section{References}

1. World Health Organization. Global tuberculosis report 2019. Geneva: World Health Organization; 2019.

2. World Health Organization. Policy statement: automated real-time nucleic acid amplification technology for rapid and simultaneous detection of tuberculosis and rifampicin resistance: Xpert MTB/RIF system. Geneva: World Health Organization; 2011.

3. Seung KJ, Linton SW. The growing problem of multidrug-resistant tuberculosis in North Korea. PLoS Med 2013;10:e1001486.

4. Shin YJ, Ki M, Sung N. A new strategy for tuberculosis control in North Korea. Epidemiol Health 2015;37:e2015053.

5. Burki T. North Korea and the global fund. Lancet Infect Dis 2018;18:501.

6. Bahk J, Ezzati M, Khang YH. The life expectancy gap between North and South Korea from 1993 to 2008. Eur J Public Health 2018;28:830-5.

7. Zastrow M. North and South Korea team up to tackle TB and malaria. Nature 2018 Nov 13 [Epub]. https://doi.org/10.1038/ d41586-018-07393-y.

8. Choi CM, June JH, Kang CI, Park JT, Oh SY, Lee JB, et al. Tuberculosis among dislocated North Koreans entering Republic of Korea since 1999. J Korean Med Sci 2007;22:963-7.

9. Park DH, Choi MH, Lim AY, Shin HY. An analysis of infectious disease research trends in medical journals from North Korea. J Prev Med Public Health 2018;51:109-20.

10. Ha S, Yoon SJ, Chun BC, Kim KJ, Roh SY, Lee EJ, et al. Biblio- 
graphical characteristics of North Korean medical journals and articles. J Korean Med Sci 2018;33:e185.

11. World Health Organization. Guidelines for the programmatic management of drug-resistant tuberculosis, WHO/HTM/ TB/2008.402. Geneva: World Health Organization; 2008.

12. Kim J, Kim D, Lee K. Study on the incidence of drug resistance according to the duration of DOTS drugs. Prev Med 2007;1:27.

13. Hong ST. North Korean medical Journals in the Galapagos. J Korean Med Sci 2018;33:e209.

14. World Health Organization. WHO treatment guidelines for drugresistant tuberculosis, WHO/HTM/TB/2016.04. Geneva: World Health Organization; 2016.

15. Joint Monitoring Mission. Tuberculosis Control Programme
Democratic People's Republic of Korea. Geneva: Joint Monitoring Mission, World Health Organziation; 2017.

16. Gee A. USA and North Korea work together on tuberculosis. Lancet 2010;375:1862.

17. Parry J. North Korea's fight against tuberculosis gets a boost. BMJ 2010;340:c2223.

18. Stone R. North Korea. New tuberculosis lab hailed as breakthrough in health diplomacy. Science 2010;327:1312-3.

19. Lee H, Ahn DY, Choi S, Kim Y, Choi H, Park SM. The role of major donors in health aid to the Democratic People's Republic of Korea. J Prev Med Public Health 2013;46:118-26.

20. Seung KJ, Franke M, Linton SW. Multidrug-resistant tuberculosis treatment in North Korea: is scale-up possible? PLoS Med 2016;13:e1002062. 